\title{
Research on Network Digital Image Information Public Opinion Incident Prevention
}

\author{
Junliu Zhong1, a, Yanfen Gan², b, Janson Young ${ }^{2, ~ c}$ and Lian Huang ${ }^{1, d}$ \\ ${ }^{1}$ School of Information Engineering, Guangdong Mechanical \& Electrical College, Guangzhou \\ 510550, China; \\ ${ }^{2}$ School of Information Science and Technology, Guangdong University of Foreign Studies South \\ China Business College, Guangzhou 510545, China; \\ ${ }^{3}$ Shool of computers, Guangdong University of Technology, Guangzhou, 510006, China. \\ aJunliuzhong@foxmail.com, bFannygyf@foxmail.com, JJansonyoung@foxmail.com, \\ dramram@126.com
}

\begin{abstract}
In recent years, the proportion of image information has raise to $90 \%$ in information transmission. With the rapid development, the network digital image also faced a series of problems. Network digital image forgery has become one of the most serious problems in the process of transmission. Image forgery has seriously affected the authenticity of the news, made the people produce panic intensified social contradictions, and triggered a wide range of public opinion problem. This has led to social disharmony and instability yet. Therefore, research on authenticity identifying of the network news images and the prevention of public opinion incident have important practical significance. In this paper, the forgery manners and reasons of the network digital images are classified in detail. We summarized the common identification methods, and put forward our own opinions on prevention strategies of public opinion incident.
\end{abstract}

Keywords: Network digital image; Prevention; Public opinion incident; Forgery.

\section{Introduction}

Network public opinion is an important form of social public opinion which refers to different views about the popular social problem in internet. It is the expression way for comments and opinions of the public. The public focus issues or hot spot news in real life. They are deeply influenced by some tendentious accounts and make some tendentious and bias speeches in Internet. Network public opinion monitoring systems [1], making use of search engine technology and network information mining technology in decision-making have been highly valued. The systems provide overall date support for decision maker to grasp public opinion dynamics in the round and make the right public opinion guidance via collecting and processing automatically by the web-page content, filtering sensitive word, aggregating subject and statistical analysis to form the public opinion brief, public opinion report and analysis report. Since 2009, engineers of Google Co. LTD published an article in journal nature about monitoring results based on search data of influenza incidence. The research based on the network data about monitoring and predicting social and economic behavior have gradually become a hot spot. People are more and more used to share information and express their appeals through Weibo, We Chat, BBS and other social networking platform. The amount of daily transmitted data on these platforms has become tens or even one hundred billion. Collecting these data for government to dynamic monitor the social public opinion is of great significance. Compared with traditional monitoring methods, the data has characteristics of strong real-time property, wide coverage and diversified information type, help the government fast, efficiently and accurately formulating policy strategy, and play a crucial role to social governance and information security on a macro level. In the monitoring and early-warning research of network public opinion security technology, Du [3] discussed the system structure of information mining of network public opinion, presented different mining technology based on the development stage of public opinion theory. Wan [4] presented a research method using the evolution volatility of the network public opinion information based on GARCH class models. Through using the major search engine to search 
a hot issue, the amount of Web pages is obtained. And then the evolution tendency of relevant public opinion of the hot issue could be analyzed. Lan [5] presented the study of Internet public opinion emergency mechanism and evolution law of safety assessment index system of Internet public opinion emergency. Autonomy [2] employed the keyword-driven for network public opinion monitoring analysis system of the public opinion monitoring system, and focused on the semantic analysis. He constructed the 3D system index from the netizens' reaction, the information characteristics of emergency and the situation spreading of emergency. In addition, the clustered analysis method of text categorization, the clustering analysis method of text dynamic, the average clustering algorithm research, etc., all focused on application's technology level. At present, the network public opinion information system has certain advantages for grasping text data. From the former studies, the research of identifying, tracking and controlling bad network information and false information are limited in the text. Internet data type is numerous and the amount of data is huge. Collecting and analyzing network digital images are more difficulty and the technology is more complex than text. So few researches are pay attention to preventing and controlling the network digital image public opinion, especially on preventing the network images public opinion incident.

The main problem of information security of network digital image public opinion is that the large number of false images information seriously affects the network society. When something was reported, the social populaces do not know beginning, development and the end of the event. They merely use their own subjective consciousness to guess the expressive comments from the texts or images of Internet, and lack of enough real information to make rational decisions and judgments. In the network media, images can enrich news content, and improve the credibility of the information and enhance propagandistic effect. When a series of false images was exposed, public opinion doubted the authenticity of the images and the media credibility. The audience began to dot not believe the information of media. Even worse, the credibility of the authoritative media could be impacted, and the honest tower built before centuries become dangerous. Therefore, the Internet public opinion incident has to be well monitored. Monitoring and preventing the network public opinion can prevent a wide range of public opinion from occurring, which are able to prevent people come into being panic emotion and avoid possible escalation of social conflicts, and keep the social stabilization and harmony.

\section{The digital image forgery manners and causes on internet}

With the development of computer and digital medium technology, the efficiency of photographing, processing, transmitting is greatly improved. It has injected new vitality to the Internet. The development of new technology provides the convenience for manipulating photos which produces some tampering events and added some doubts on the reality of network images for people. How to deal with the challenge of new technology for network image authenticity becomes the focus of discussion. At present, the processing technic can be concluded as the following: (1) the false news images with words, cut out a part of image or distort the images information expression, make a lot of people mislead. (2) Copying image content and pasting it into other image, or copying and adjusting interested region and pasting it into another part of the same image to achieve a certain desired effect. In 2005, A works winning natural and environmental news gold prize of the 1st China International Press Photo Contest (CHIPP), "pigeon living in square were injected influenza vaccine", is the typical image composited case. (3) Using photography technology or posing to design some inexistent scene generates illusion, such as the event of "Zhou Tiger".

With the development of media technology, media platforms become diversity. And the competition among the medium is increasingly fierce, so that the environment of the media companies are worsening. In this external environment, the medium actively make fake news events or tamper news images for attracting more reader's attention, such as the cardboard bun event. To chase the interests, media would always cater to the reader's flavors, therefore the tampering images 
are increasingly presented. At present, the imperfect news management system and the unestablished press law help some news forgers escaping punishment.

\section{Identification of network forgery image and the strategies of public opinion incident}

For the network forgery images, we usually identify by the following methods [6]. (1)For some forgery images, scientists presented some algorithms to automatically identify forgery images. The algorithms mainly make use of the forgery feature to identify images. (2) When modifying images, they firstly enlarge the image to tamper. This operation may lead to obvious marks between cohesion places, so we can first enlarge the image before identify the forgery images by obvious marks. (3) Based on the image information, background knowledge and common sense of life, we should apply logical relation and inference for finding out the contradiction to overthrow the authenticity of the image. These methods to discern between true and false are employed after images releasing or public opinion incidents triggering. If we want to prevent public opinion incident, we have to monitor its source.

Now, Internet image and video data accounted for $90 \%$ of the whole internet data [7]. The real-time monitoring is more difficulty, but we can try to prevent the public opinion happening. We can identify network image information when uploading or downloading. For identifying digital image, we should understand various methods for the digital image authentication. The authentication methods can be divided into two categories: one kind is the digital watermarking and the digital signature for image, which belong to the active authentication. This authentication needs to add the watermark or auxiliary information to image in advance. In fact, not all the images can be added information in advance; the other category is passive authentication, which is not depends on any pre-signature extraction or pre-embedding information in advance. This category according to the image itself can be judged whether it has been forged, such as synthesis, retouching.

According to the above two kinds of authentication, we put forward the preventing strategies about digital image public opinion information in internet:

(1) For the government, enterprises and institutions and scientific research institutions in the intranet, they widely used the PDF and JPG file type. This kind of image file will be used as the unit interior circulated browsing, therefore, we can adopt active authentication to embed watermark information in electronic file before transmitting. This embedding information are known for the sender and receiver, so the receiver can extract image watermark, check certification and identify authenticity after receiving digital image in network.

(2) For the network images uploaded by individual, this kind of image is not suitable for applying active authentication technology. It is pressing need a prevention system model which is based on passive authentication. This technique can detect an uploading image without embedding watermark, which can prevent image from illegally and maliciously transmitting.

These two strategies described above can detect most of forged image before the image transmission. These strategies can play an important role of preventing public opinion incident.

\section{Conclusion}

Internet digital image forgery can cause a wide range of Internet public opinion incidents. Research of preventing and monitoring is a fused subject among journalism, communication and computer science. It has strong practicality and deep theory. The flourish of network news image is promoted by the combination of news image and network. Behind the prosperity, the flourish hid deep crises. A series of ethical problem have been brought by the image transmission, so the problem has been attracted the journalism and communication's attention at present. A lot of scholars have carried out extensive researches. These researches do not form complete theoretical system yet. It is urgent need to expand the breadth and depth of research. To prevent the forged images from causing public opinion incidents, we have to monitor these images in advance, which should combine with the 
current advanced computer anti-counterfeiting technology. That will be an important research direction about social science interdisciplinary in the future

\section{Acknowledgments}

This work is supported by the 2016 Guangzhou philosophy and social science "Thirteen Five" project - Digital image forgery cause public opinion incident prevention countermeasures and technical research based on internet information security (No.2016gzqn23).

\section{References}

[1] Zhang Yue, Luo Ping, Liu Qianli. A Study in Monitoring of Network Public Opinion Based on Life Cycle Theory. Information Science, Vol.31 (2013), No.11, p.22-25.

[2] Autonomy, Safety and Monitoring. 2014-11-20. http://www.autonomy.com.cn/content/solutions/security_survellance,zh,html.

[3] Du Aning, Cheng Xiaoming. Frequent items maintaining algorithms in network traffic analysis. Journal on Communications. Vol.27 (2006) No.2, p.9-15.

[4] Wan Yuan. Research on Mining of Internet Public Opinion Based on Semantic and Statistic Analysis (Wuhan University of Technology, China, 2012), p.24-56.

[5] Lan Yuexin, On Construction of Emergency Network Safety Evaluation Index System. Information Science, Vol.30 (2011) No.7, p.73-76.

[6] Ji Minmin, In Network Transmission News Forgery Images Analyzing and Researching Countermeasure (Shandong Normal University, China, 2012).

[7] Information

on: http://www.cnnic.net.cn/hlwfzyj/hlwxzbg/hlwtjbg/201507/t20150722_52624.htm. 